\title{
An Efficient Method for Calculating the Absorption Enhancement in Solar Cells with Integrated Plasmonic and Photonic Nanoparticles
}

\author{
P. Manley ${ }^{1,2}$, F. Schmidt ${ }^{2}$ and M. Schmid ${ }^{1,3}$ \\ ${ }^{1}$ Helmholtz Zentrum Berlin für Materialien und Energie GmbH, NanooptiX, Hahn-Meitner-Platz 1, 14109, \\ Berlin, Germany \\ ${ }^{2}$ Zuse Institute Berlin, Takustrasse 7, 14195, Berlin, Germany \\ ${ }^{3}$ Freie Universität Berlin, Department of Physics, Arnimallee 14, 14195, Berlin, Germany \\ phillip.manley@helmholtz-berlin.de
}

\begin{abstract}
We present a method for calculating the plasmonic and photonic enhancement of the absorption in solar cells. The method involves coupling between a transfer matrix method to describe light propagation in the layered stack and Mie theory for calculating the absorption and angular scattered field distribution from the nanoparticles. We also compare the method to rigorous simulations.
\end{abstract}

\section{INTRODUCTION}

Due to cost pressures or material limitations, many solar cell devices are required to be as thin as possible. One such example is that of chalcopyrite solar cells with an absorber layer consisting of $\mathrm{Cu}\left(\operatorname{In}_{x}, \mathrm{Ga}_{x-1}\right) \mathrm{Se}_{2}$. Due to this material containing the rare element In, it is desirable to reduce the thickness of this layer. This can be detrimental to the complete absorption of light by the solar cell, necessitating a light management concept in order to retain a high absorption of incident light. A promising concept for light management is the use of plasmonically active nanoparticles integrated into the absorbing layer of the solar cell [1]. These can positively influence the absorption in two ways: the scattering from the particles into large angles will increase the optical path length in the absorbing layer; secondly the localization of the electromagnetic field around the nanoparticle can lead to increased absorption. In order to determine the optimum use of plasmonically active nanoparticles, simulations can be used to predict which values of nanoparticle size, and which kind of material, coating etc. provide a high absorption enhancement. Currently simulations typically rely on rigorous methods which provide a high degree of accuracy with a correspondingly high computational cost. We present a computationally efficient method based on the coupling between the transfer matrix method for layered stack propagation, and Mie theory to describe the absorption and angular scattered field distribution due to the nanoparticles.

\section{THEORETICAL BACKGROUND}

For a layered stack of homogeneous materials, the propagation of light in the layer can be efficiently computed using the transfer matrix (TM) method. Using the method, both the propagation of a forwards and backwards traveling wave inside of each layer, and the reflection and transmission at each interface are determined by a 2 $\times 2$ matrix. The global solution is then determined by multiplying together all the individual matrices to obtain propagation through the entire stack. For more details we refer the reader to [2].

Mie theory is an analyitical solution to Maxwell's equations for a sphere in a homogeneous medium illuminated by a plane wave. This gives the electromagnetic field both inside and outside the sphere at all distances from the sphere. For more information see [3]-[4].

Fig. 1 shows examples of the capabilities of Mie theory. Part (a) shows the scattering cross section of a Ag nanoparticle embedded in chalcopyrite material over a large range in both wavelength and particle diameter. This can be calculated in seconds using Mie theory. The highest scattering in this case is given between 700 and 800 $\mathrm{nm}$ wavelength by a particle diameter of between 40 and $60 \mathrm{~nm}$. However, considering that the resonance becomes more broadband for larger sphere diameters, a larger sphere size may be more appropriate when considering 


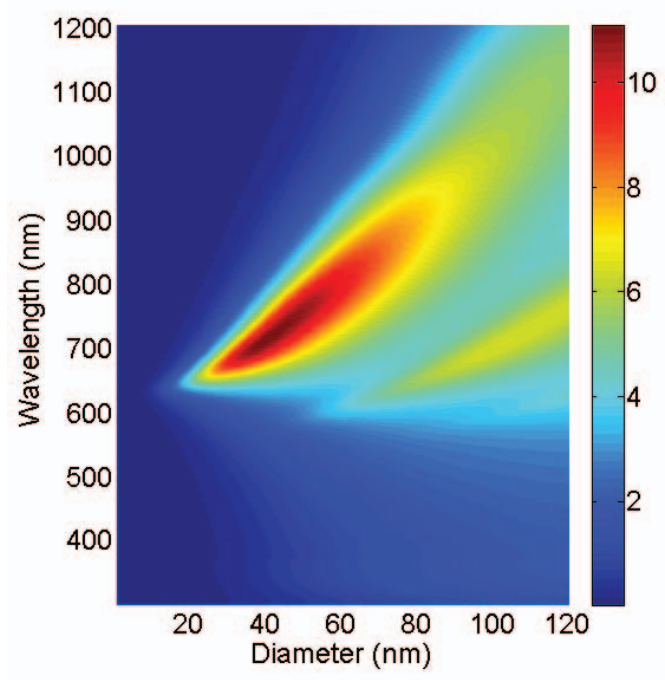

(a)

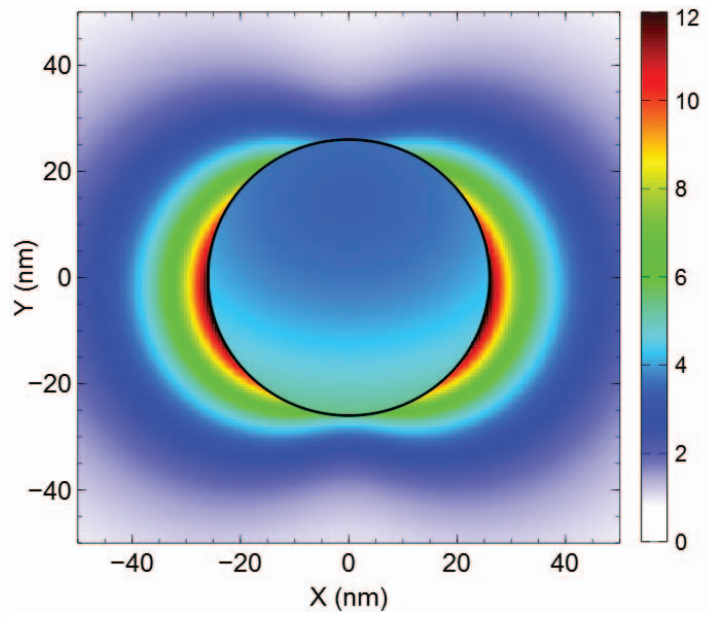

(b)

Fig. 1: (a): The scattering cross section for a Ag nanoparticle embedded in chalcopyrite material calculated with Mie theory. (b): The near field calculated around a $\mathrm{Ag}$ nanoparticle embedded into chalcopyrite material with a diameter $50 \mathrm{~nm}$ at a wavelength of $750 \mathrm{~nm}$.

that an absorption enhancement may be required over a wide range of wavelengths. Part (b) shows the electric field distribution around the nanoparticle for the peak scattering cross section intensity of diameter $50 \mathrm{~nm}$ and wavelength $700 \mathrm{~nm}$.

Currently, integrated plasmonic solar cells are simulated using rigorous methods such as the finite element method (FEM). The FEM allows for a lot of flexibility in the geometry of the simulation, which can more accuratly model a solar cell with integrated plasmonics. However the method comes with significant computational costs, meaning that an optimised system would be very time consuming to determine.

\section{RESUltS AND DiscusSiON}

In the transfer matrix algorithm the propagation of light inside each layer is described by a plane wave, this can also be used as the input to a Mie theory calculation which is valid for an incident plane wave. Mie theory describes the outgoing wave as a combination of vector spherical harmonics, which for a sufficient distance from the source (i.e. in the far field) describe a spherical plane wave. A spherical plane wave can be decomposed into plane waves propagating at different angles, which in turn can be used as the input for transfer matrix calculations. Thus the two methods may be coupled together. Previous attempts have been made to include the effects of nanoparticle scattering into a transfer matrix scheme [5]. However this was limited to the dipole approximation and did not include near field absorption.

Light inside the multilayer stack can interact multiple times with an integrated nanoparticle necessitating an iterative approach. The fields are proagated inside the the layered stack and the Mie theory layer multiple times until the amount of power transfered between the two domains is sufficiently small, indicating that all of the incident power has been reflected, transmitted or absorbed. A schematic for the computational system used is given in fig. 2(a).

Although the aforementioned coupled approach will be able to give quantitative predictions for the absorption enhancement, a simpler approach can give us a qualitative understanding. Fig. 2(b) shows the absorption in a solar cell with a $500 \mathrm{~nm}$ absorber layer and without nanoparticles (dashed line) calculated using the TM model. Also plotted is the scattering cross section for a $100 \mathrm{~nm}$ diameter Ag nanoparticle embedded into the solar cell (dotted line) calculated using Mie Theory. By looking at the spectral overlap between the nanoparticle resonance and the region of poor solar cell absorption, we can begin to optimise the particle size for efficient absorption 


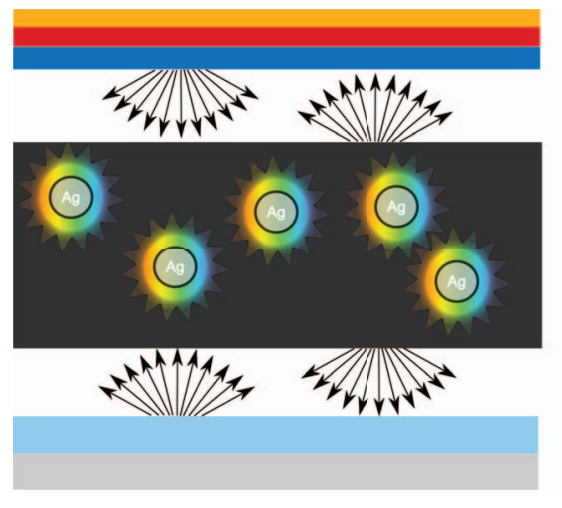

(a)

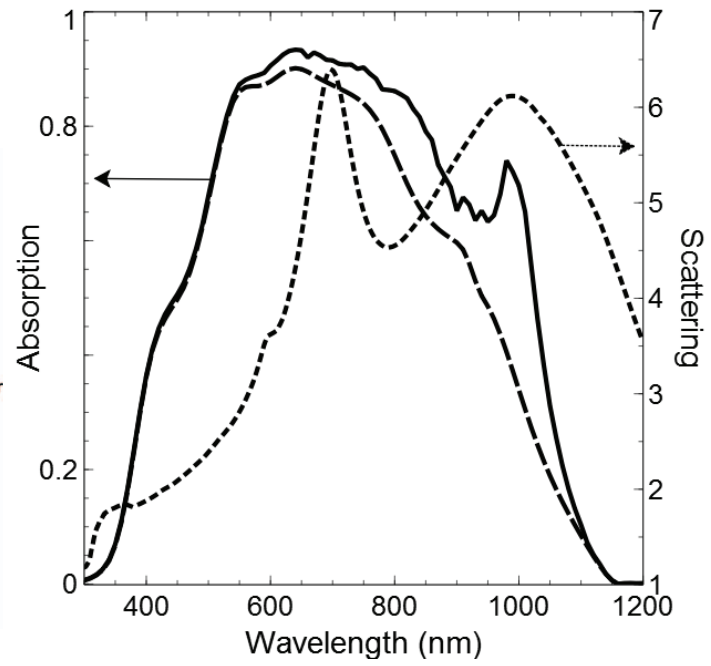

(b)

Fig. 2: (a) Schematic of the simulation procedure for coupling together transfer matrix and Mie theory calculations. (b) Simulated absorption in the chalcopyrite layer of a solar cell. The reference absorption (dashed line) is calculated via the transfer matrix. The plasmonically enhanced absorption (solid line) is calculated via the finite element method.

enhancement. Note that the absorption corresponds to the left axis whilst the scattering cross section corresponds to the right axis, as shown by the arrows in the figure. Also shown is the absorption enhancement calculated using FEM simulations for a $500 \mathrm{~nm}$ thickness absorber layer with an integrated $100 \mathrm{~nm}$ diameter Ag nanoparticle. The Absorption enhancement compared to without the nanoparticles corresponds well to the spectral regions of high scattering predicted by Mie theory, justifying the simplified approach. However a quantitative prediction using the combination of the TM method and Mie theory would allow for a true fast efficient optimisation of the nanoparticle properties.

\section{CONCLUSION}

We have presented a fast and efficient method for calculating the propagation of light inside a layered stack containing plasmonic nanoparticles. This allows the absorption enchancement in solar cells with integrated nanoparticles to be rapidly optimised. The method is compared to the results obtained using rigorous methods to assess the accuracy.

\section{ACKNOWLEDGEMENT}

The authors would like to acknowledge funding by the DFG (German Research Foundation) in the DFG research center MATHEON and support from the Initiative and Networking fund of the Helmholtz Association for the Young Investigator Group VH-NG-928.

\section{REFERENCES}

[1] K.R. Catchpole, and A. Polman, "Plasmonic Solar Cells", Optics Express, 16(26), pp. 21793-21800, 2008

[2] P. Manley, G. Yin, and M. Schmid, "A method for calculating the complex refractive index of inhomogeneous thin films", Journal of Physics D: Applied Physics, 47(20), pp. 205301, 2014

[3] C.F Bohren, and D.R. Huffmann, "Absorption and Scattering of Light by Small Particles", 1998

[4] M. Schmid, P. Andrae, and P. Manley, "Plasmonic and photonic scattering and near fields of nanoparticles", Nanoscale Research Letters, 9, pp. 50/51-50/59, 2014

[5] M. Schmid, R. Klenk, M.C. Lux-Steiner, M. Topic, and J. Krc, "Modeling plasmonic scattering combined with thin-film optics", Nanotechnology, 22(2), pp. 025204/025201-025204/025210, 2011 